\title{
A NEW EVOLUTIONARY INTERPRETATION OF THE IRAS TWO-COLOUR DIAGRAM
}

\author{
P. GARCÍA-LARIO \\ LAEFF - Estación de Villafranca del Castillo. Apdo. 50727. E-28080 Madrid (Spain) \\ A. MANCHADO \\ Instituto de Astrofísica de Canarias. E-38200, La Laguna, Tenerife (Spain) \\ and \\ S.R. POTTASCH \\ Kapteyn Laboratorium, Postbus 800. NL-9700, AV Groningen (The Netherlands)
}

\begin{abstract}
A new evolutionary interpretation of the sequence of colours observed in the IRAS two-colour diagram by AGB and post-AGB stars is given, which is capable of explaining the observational properties of both kind of objects. It is useful to define a parameter $\lambda$ to define the position of a given star in this "infrared main sequence" (IRMS). Adopting
\end{abstract}

$$
[12]-[25]=\frac{1}{1.096} \operatorname{Ln} \lambda \quad[25]-[60]=-2.42+0.72 \lambda
$$

and from the analysis of the expansion velocities, mass loss rates and luminosities observed in a selected sample of non-variable OH/IR stars with no optical counterpart in the Galactic bulge as a function of $\lambda$, we conclude that the position in the IRAS two-colour diagram at which a star leaves the IRMS $\left(\lambda_{\max }\right)$ only depends on the initial mass $M_{i}$ of the progenitor star, so that only massive objects can reach the upper end of this sequence. The relation found is:

$$
\lambda_{\text {max }}=3.2 \log \frac{M_{i}}{M_{\odot}}+2.45
$$

Expansion velocities increase with the initial mass while every point in the IRMS is found to be associated to a certain value of the mass loss rate. This model also predicts the evolution with time of the mass loss rate during the AGB as a function of the initial mass of the progenitor star, and confirms that most known planetary nebulae are the result of the evolution of considerably massive stars (between 2-3 solar masses) which means that the contribution of processed material to the interstellar medium is considerably higher than what theoretical models predict. Type I PNe are the result of the evolution of $3-5 M_{\odot}$ progenitors while progenitors with $M_{i} \leq 1.2 M_{\odot}$ probably do not give PNe. The model is also in agreement with the narrow distribution of core masses found in central stars of $\mathrm{PNe}$ and white dwarfs and with the usual expansion velocities found in $\mathrm{OH} / \mathrm{IR}$ stars. 